\title{
Negative Resistance and Its Impact on a RC-DC Driven Electric Circuit
}

\author{
Haiduke Sarafian \\ The Pennsylvania State University, University College, York, USA \\ Email: Has2@psu.edu
}

How to cite this paper: Sarafian, H. (2021) Negative Resistance and Its Impact on a RC-DC Driven Electric Circuit. Journal of Electromagnetic Analysis and Applications, 13, 103-110.

https://doi.org/10.4236/jemaa.2021.137007

Received: July 5, 2021

Accepted: July 28, 2021

Published: July 31, 2021

Copyright $\odot 2021$ by author(s) and Scientific Research Publishing Inc. This work is licensed under the Creative Commons Attribution International License (CC BY 4.0).

http://creativecommons.org/licenses/by/4.0/

(c) (i) Open Access

\begin{abstract}
It is a common misconception that electric "resistance" always is a positive defined electric element. i.e., the plot of the voltage across the resistor, $\mathrm{V}$ vs. its current, $i$ is a slanted straight line with a positive slope. Esaki diode also known as tunnel diode is an exception to this character. For a certain voltage range, the current recedes resulting in a line with a negative slope; it is interpreted as negative resistance. In this research flavored report, we investigate the impact of the negative resistance in a typical classic electric circuit. E.g., a tunnel diode, $\mathrm{D}$ is inserted in a classic electric circuit that is composed of an ohmic resistor, $\mathrm{R}$ and a capacitor, $\mathrm{C}$ which are all in series with a $\mathrm{DC}$ power supply. The circuit equation for the RCD circuit is a nonlinear ordinary differential equation (NLODE). In line with the ever-growing popular Computer Algebra System (CAS), this is solved numerically utilizing two distinctly different CASs. The consistency of the solutions confidently leads to the understanding of the impact of the negative resistance. The circuit characteristics are compared to the classic analogous RC circuit. The report embodies an atlas of characteristics of the circuits making the analysis visually comprehensible.
\end{abstract}

\section{Keywords}

Tunnel Diode, Negative Electric Resistance, Computer Algebra System, Mathematica, Maple

\section{Introduction}

We begin with the well-known characteristics of an ohmic resistor. Denoting V, the voltage across the resistor and $i$, its current plot of these two variables is depicted in Figure 1.

The horizontal axis, $\mathrm{V}$ is the voltage applied to the ends of a resistor, the vertical axis, $i$ is the current through the resistor. As shown, the relationship be- 
tween these variables across the entire applied voltage is linear as shown by a slanted straight line. Its constant positive slope equals, $1 / \mathrm{R}$ where $\mathrm{R}$ is the ohmic resistor. This is known as Ohm's law, c.f. [1]. The majority of the metallic conductors are subject to this law. As a practical application, its positive value for a conductor with resistivity, $\rho$ length, $\ell$ and a constant cross-section, $A$ is formulated as, $R=\rho \ell / A$, c.f. [1]. Naively if one begins with the latter formula, as is being done in college texts c.f. [1] naturally, it excludes the potential of being negative. In contrast to what is discussed consider a hypothetical scenario where $\mathrm{i}-\mathrm{V}$ character is subject to shown in Figure 2.

As depicted, the i-V diagram has three segments. The first and the third segments are slanted straight lines with positive slopes, same as Figure 1 with the same physics interpretation. The mid-segment, however, although is a slanted straight line has a negative slope! Justifiably, it has to be interpreted as negative resistance! In general, the profile characteristics of most of the physical phenomena are smooth and continuous, Figure 2 ought to be smoothened. As such the discontinuities are rounded in Figure 3.

For the sake of comparison collectively the last three figures are shown in Figure 4.

With this background the question is: "Is there a physical evidence attesting to the actual existence of an element exhibiting characteristics shown in Figure 3?" The answer is YES. Esaki, aka tunnel diode is one such element [2]. A workstation utilizing a tunnel diode was setup at our Electrical Engineering Laboratory. Figure 5 is the photo of the actual setup. In addition to the tunnel diode various

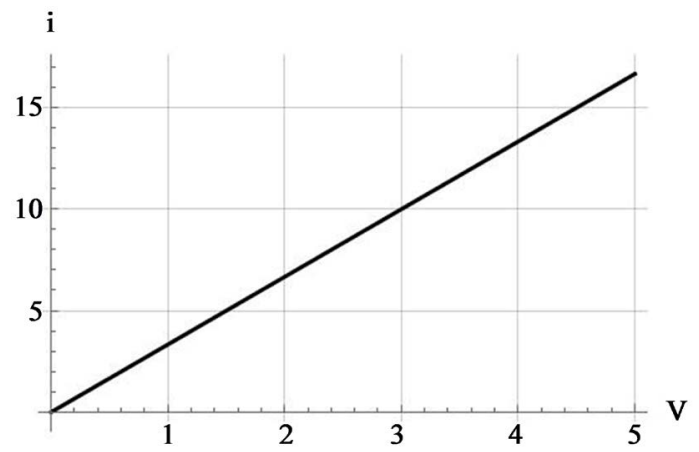

Figure 1. i-V diagram of an ohmic resistor.

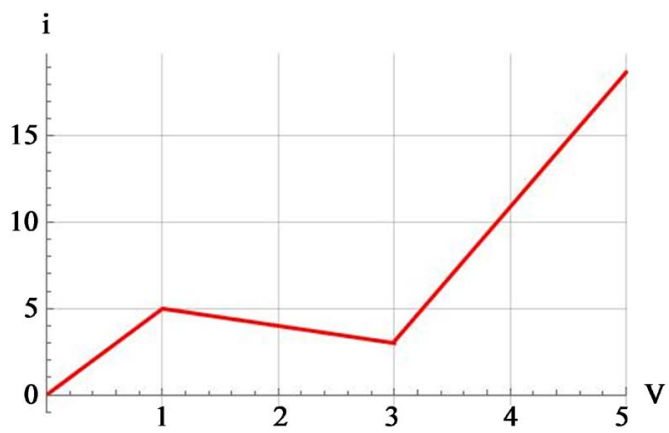

Figure 2. i-V diagram of a hypothetical element exhibiting a negative slope. 


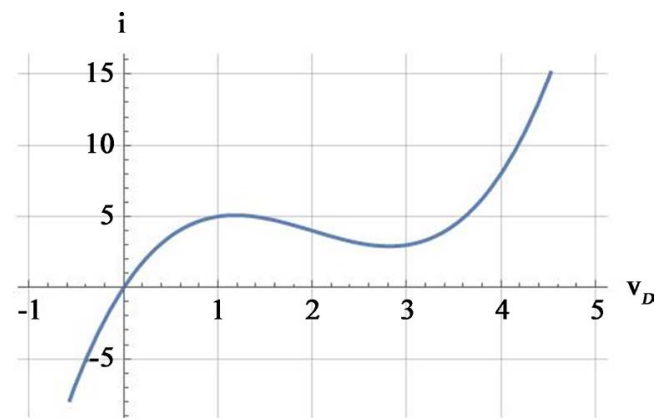

Figure 3. Smoothened version of Figure 2.
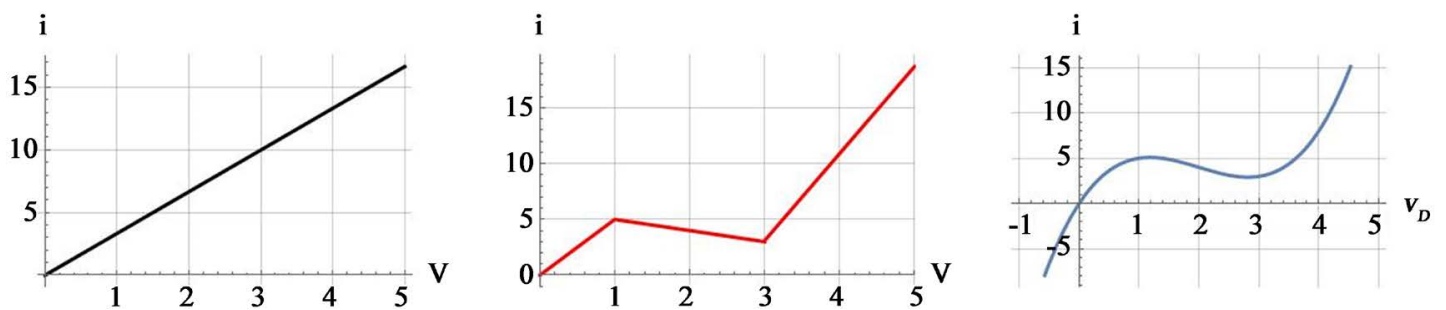

Figure 4. Description of individual graph is the same as Figures 1-3.
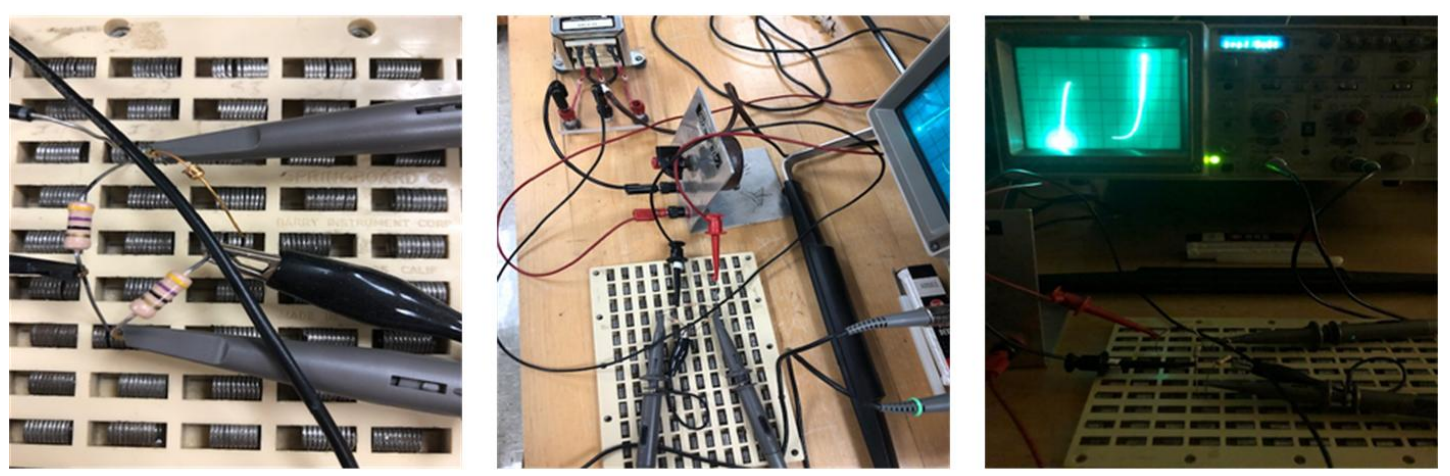

Figure 5. The two left images are the breadboard circuits. The shiny gold-colored element between the two color-coded resistors in the left image is the tunnel diode. The snapshot of the $\mathrm{i}-\mathrm{V}$ signal of the diode is captured on the Scope shown on the far-right image.

other components are shown as well. The voltage across the diode and its current are displayed on the Scope, the far-right image.

As shown the snapshot of the $\mathrm{i}-\mathrm{V}$ signal resembles Figure 3; it has three segments. The first and the third segments on the $\mathrm{i}-\mathrm{V}$ Scope are slanted and linear with positive slopes. Within these volage ranges, the diode acts as an ohmic resistor. Interpolate the edges of the latter segments resulting in a slanted straight line with a negative slope, indicative of negative resistance.

With this background information and the factual existence of negative resistance, the physics question is: "What is the impact of a negative resistance in the characteristics of a typical classic electric circuit?" Among many classic circuits, we consider an RC circuit and insert a tunnel diode all in series with a DC power supply, making the circuit RCD-series DC driven circuit. Schematic of the circuit is shown in Figure 6. 


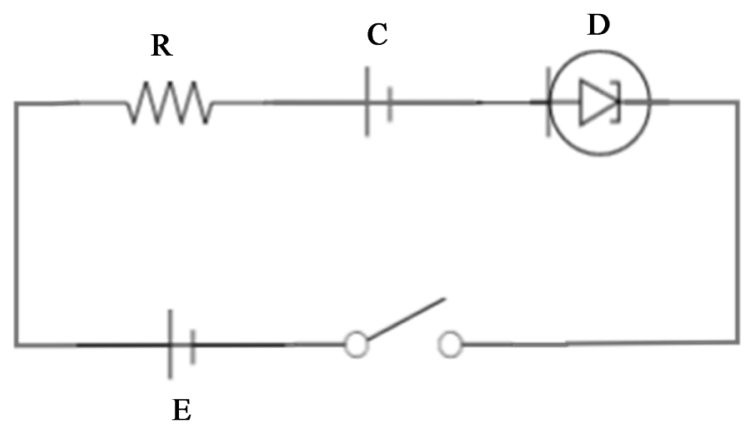

Figure 6. Three elements: A resistor, $\mathrm{R}$ a capacitor, $\mathrm{C}$ and a tunnel diode, $\mathrm{D}$ are in series with a DC power supply.

With these objectives we craft a report addressing the issues of interest. This report has three main sections. In addition to Sect 1, Introduction, in Sect 2, Circuit Analysis, formulation of the problem including the circuit equation depicted in Figure 6 is presented. Solution of the nonlinear circuit equation is presented graphically as well. Characteristics of the circuit are compared to the analogous classic circuit highlighting the differences. This section also includes an atlas of complementary graphic information assisting the understanding of the issues of interest. The last section of the report, Conclusions, addresses summary of the achieved results.

\section{Circuit Analysis}

We begin with the characteristics of the tunnel diode. Guided with the displayed Scope signal shown in Figure 5, a functional relationship between the voltage applied to the ends of the diode, $V_{D}$ and the running current, $i$ is parametrized in (1).

$$
i=\alpha-\beta\left(V_{D}-\delta\right)+\gamma\left(V_{D}-\delta\right)^{3}
$$

This is an odd-ordered function with respect to the voltage, $V_{D^{\circ}}$ A cubic function is needed to produce the shown signal in Figure 5. A similar analytic function is used in [3]. Both $i$ and $V_{D}$ are implicit time dependent functions. Equation (1) has four time-independent parameters: $\{\alpha, \beta, \gamma, \delta\}$. By adjusting these values, one adjusts the coordinates of the extremum, the separation distance between them, the coordinate of the inflection as needed. One set of such parameters are stored in listing values1. Furthermore, as shown on the Scope as well as Figure 4 the current and voltage initially are subject to $i=V_{D}=0$. Applying this to (1) relates the constant parameters reducing the degrees of freedom, namely,

$$
\begin{gathered}
\text { values } 1=\{\alpha->4 ., \beta->2 ., \delta->2 ., c->1 .\} ; \\
\gamma=\frac{\alpha+\beta \delta}{\delta^{3}}
\end{gathered}
$$

Utilizing these values, plot of (1) is shown on the far-right graph of Figure 4.

The schematic of the circuit is shown in Figure 6. All three elements and the 
DC power supply are in series. Applying the loop theorem, i.e., Kirchhoff's 1st law yields c.f. [1],

$$
E-V_{C}=V_{R}+V_{D}
$$

Substituting for the $V_{c}$ and $V_{R}$, we get,

$$
E-\frac{1}{c} q(t)=R i(t)+V_{D}(t)
$$

The $i(t)$ and $V_{D}(t)$ are related via (1). To follow the standard method solving (4), one might wish by solving (1) replacing $V_{D}(t)$ in terms of $i(t)$ resulting in an $i(t)$ dependent equation. However, this is not a viable option because (1) is a cubic equation. An alternative solution is to differentiate both sides of (4) with respect to $\mathrm{t}$ and then replace $\frac{\mathrm{d}}{\mathrm{d} t} q(t)$ with $i(t)$. This requires an intermediate step calculating $\frac{\mathrm{d}}{\mathrm{d} t} i(t)$, that is carried out utilizing (1). After some algebraic simplifications, we arrive at,

$$
\begin{aligned}
& \left\{R\left[-\beta+3 \gamma\left(V_{D}(t)-\delta\right)^{2}\right]+1\right\} \frac{\mathrm{d}}{\mathrm{d} t} V_{D}(t) \\
& +\frac{1}{c}\left[\alpha-\beta\left(V_{D}(t)-\delta\right)+\gamma\left(V_{D}(t)-\delta\right)^{3}\right]=0
\end{aligned}
$$

This is the circuit equation, where $\gamma$ is subject to (2). This is a first order nonlinear ordinary differential equation (NLODE). Presumably, the solution of (5) yields $V_{D}(t)$. This solution in hand yields the rest of the characteristics of the circuit, e.g., the current, $i(t)$. However, longhand analytic calculation solving (5) fails, so did symbolic calculation of two different CASs, Mathematica [4] and Maplesoft [5]. Equation (5) is solved numerically. As mentioned in the Abstract, both CASs are used. The solutions are consistent and are the same.

Guided by the plot of $i$ vs. $V_{D}$ i.e., the right most graph of Figure 4, one realizes that there is a voltage range where the diode exhibits negative resistance. To activate i.e., to reach this voltage range the voltage of the power supply, $E$ is adjusted accordingly. Noting this (5) is solved for a wide range of emfs. The results are shown systematically by a set of plots shown in Figure 7. Each graph shows two different colored curves. The vertical axis is indicative of $V_{D}$ (Blue), and the current, i (dashed Red). Both curves are plotted vs. time, $t$. By trial and error, we searched for a suitable ohmic resistor making the shown signals appropriately meaningful. As such, the value of the ohmic resistor, $R$ is set at $R=0.2 \Omega$. The title of the plots is the value of $\epsilon$, which is a fraction of the applied emf s, $E$.

In all shown plots the solid blue curves are the plots of the solution of (5), i.e., $V_{D}(t)$. With exception of the first graph that serves as the checking point, e.g., zero $\epsilon$ and hence no signal, the second plate justifies our previous observation. Meaning, because the $\epsilon$ is not high (see Figure 3 ) the voltage across the diode, $V_{D}$ is in the positive slope range and hence the circuit is a classic RC circuit with an effective ohmic resistor c.f. [1]. As such, the current (dashed red curve) after 

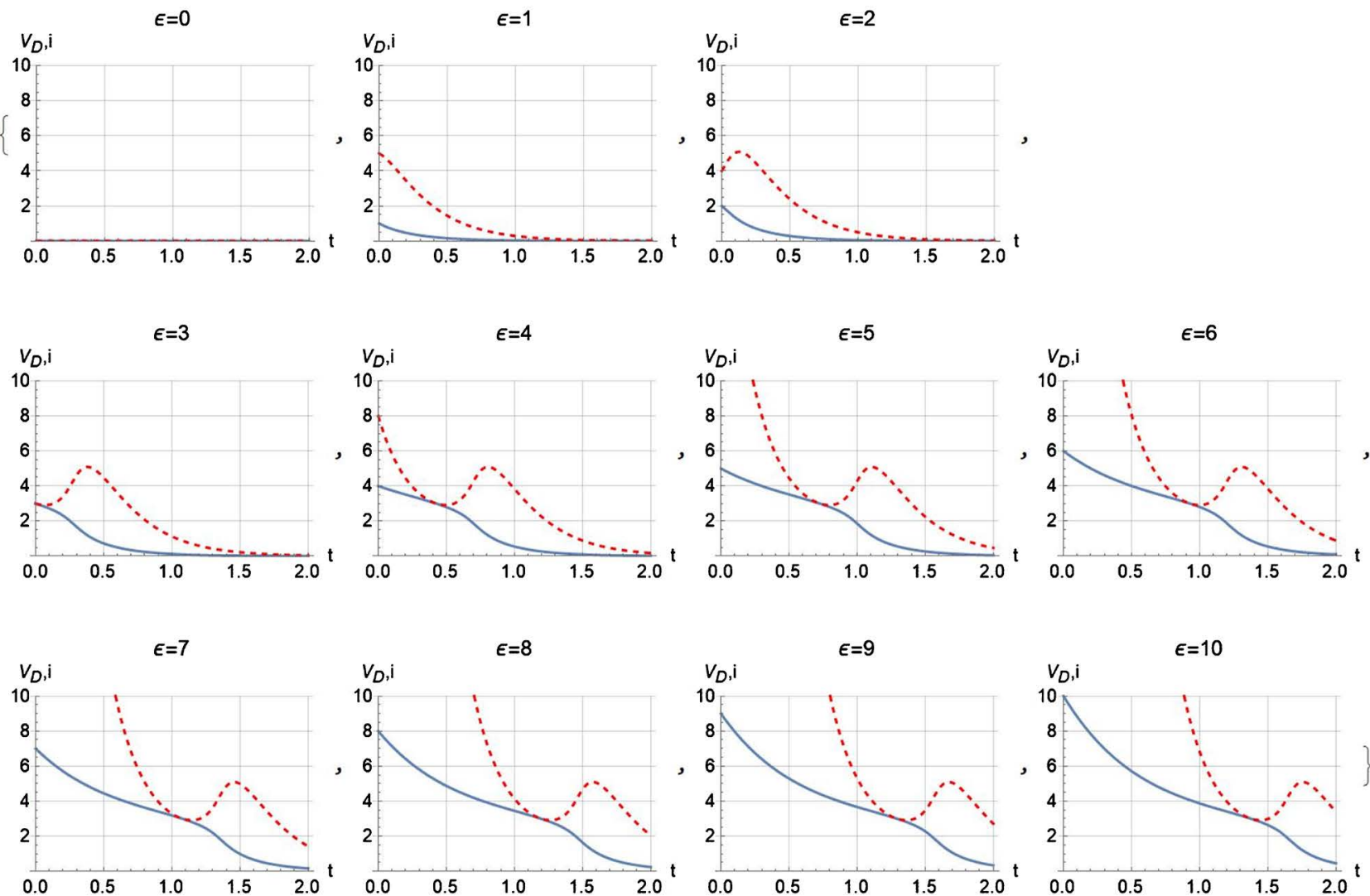

Figure 7. The voltages across the diode, $V_{D}$ and the current, $\mathrm{i}$ are shown in solid blue and dashed red vs. time, respectively. The titles are the associated $\epsilon$ 's.

$\epsilon$ (5)

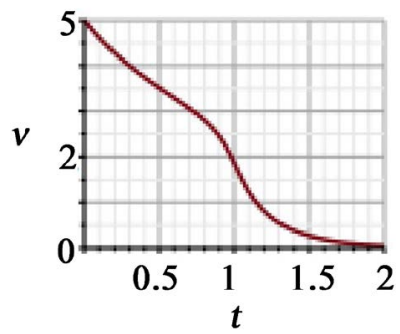

Figure 8. Plot of $V_{D}$ vs. $t$. This is the graph of solution (5) utilizing Maplesoft [5] for $\varepsilon=5$. 
i

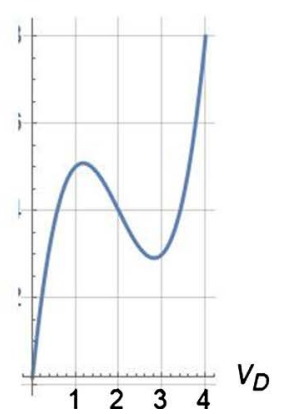

$\epsilon=4$

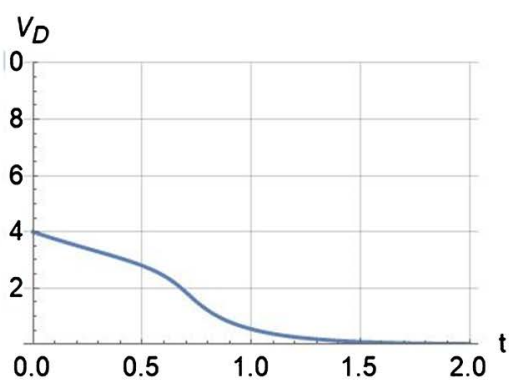

$\epsilon=4$

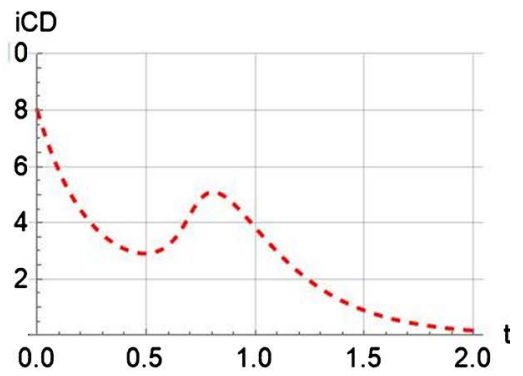

Figure 9. The middle graph is the solution of (5), the right most graph is the plot of $i(t)$. The left graph is the combined plot of the last two. Interested readers may find [6] [7] for creating graphs and programming resourceful.

of the voltage across the diode its slope and the running time, $t$. Utilizing the solution of (5) and substituting in (1) yields the explicit expression for the current as a function of time; $i=i(t)$. As noted, a gallery of these two quantities is displayed in Figure 7. Both these quantities are explicit time dependent. It is amazingly reassuring noting that by folding the time variable between these two we were able to recover the time-independent characteristics of the tunnel diode depicted in far right of Figure 4. One such case for $\varepsilon=4$ is shown in Figure 9.

\section{Conclusion}

One of the objectives of this report is to learn about the impact of negative resistance in electric circuits. As shown despite the common misconception negative resistance exists, its inclusion impacts the characteristics of classic circuits. This has not been addressed in the professional literature. Systematically we shown inserting a tunnel diode in a typical classic circuit is conducive to the analytically unsolvable circuit equation. Utilizing CASs, the latter is solved numerically. A set of comprehensive results depicting the characteristics of the circuit is included underlying the impact of the negative resistance.

\section{Acknowledgments}

The author acknowledges the John T. and Paige S. Smith Professorship funds for completing and publishing this work. He is grateful for the assistance of Professor Harley Hartman at Electric Engineering Department for elaborately setting a test-station displayed in Figure 5. He also appreciates Ms. Jamaira Unangst for her administrative support.

\section{Conflicts of Interest}

The author declares no conflicts of interest regarding the publication of this paper.

\section{References}

[1] Jackson, D. (1998) Classical Electrodynamics. 3rd Edition, John Wiley, Hoboken.

[2] Tunnel Diode. https://www.physics-and-radio-electronics.com/electronic-devices-and-circuits/sem 
iconductor-diodes/tunneldiode-howitworks.html

[3] Abarbanel, H., et al. (1993) Introduction to Nonlinear Dynamics for Physicists. World Scientific Publishing Company, New Jersey.

[4] Mathematica Vision 12.1.1. http://Wolfram.com

[5] Maple 2021. https://www.maplesoft.com

[6] Wolfram, S. (1996) Mathematica Book. 3rd Edition, Cambridge University Press, Cambridge.

[7] Sarafian, H. (2019) Mathematica Graphics Examples. 2nd Edition, Scientific Research Publishing, Wuhan. 